\title{
Elastic Remote Methods
}

\author{
K.R. Jayaram ${ }^{\star}$ \\ HP Labs, Palo Alto, CA \\ jayaramkr@hp.com
}

\begin{abstract}
For distributed applications to take full advantage of cloud computing systems, we need middleware systems that allow developers to build elasticity management components right into the applications.

This paper describes the design and implementation of ElasticRMI, a middleware system that (1) enables application developers to dynamically change the number of (server) objects available to handle remote method invocations with respect to the application's workload, without requiring major changes to clients (invokers) of remote methods, (2) enables flexible elastic scaling by allowing developers to use a combination of resource utilization metrics and fine-grained application-specific information like the properties of internal data structures to drive scaling decisions, (3) provides a high-level programming framework that handles elasticity at the level of classes and objects, masking low-level platform specific tasks (like provisioning VM images) from the developer, and (4) increases the portability of ElasticRMI applications across different private data centers/IaaS clouds through Apache Mesos [5].
\end{abstract}

Keywords: programmable elasticity, scalability, distributed objects.

\section{Introduction}

Elasticity, the key driver of cloud computing, is the ability of a distributed application to dynamically increase or decrease its use of computing resources, to preserve its performance in response to varying workloads. Elasticity can either be explicit or implicit.

Implicit vs. Explicit Elasticity. Implicit elasticity is typically associated with a specific programming framework or a Platform-as-a-Service (PaaS) cloud. Examples of frameworks providing implicit elasticity in the domain of "big data analytics" are map-reduce (Hadoop [13] and its PaaS counterpart Amazon Elastic Map Reduce [10]), Apache Pig [2], Giraph [12], etc. Implicit elasticity is handled by the PaaS implementation and is not the responsibility of the programmer. Despite being unable to support a wide variety of applications and computations, each of these systems simplifies application development and deployment, and employs distributed algorithms for elastic scaling that are optimized for its programming framework and application domain.

\footnotetext{
* Thanks to Patrick Eugster and Hans Boehm for helpful feedback.
} 
Explicit Elasticity, on the other hand, is typically associated with Infrastructureas-a-Service (IaaS) clouds and/or private data centers, which typically provide elasticity at the granularity of virtualized compute nodes (e.g., Amazon EC2) or virtualized storage (e.g., Amazon Elastic Block Store (EBS)) in a way that is agnostic of the application using these resources. It is the application developer's or the system administrator's responsibility to implement robust mechanisms to monitor the application's performance at runtime, request the addition or removal of resources, and perform load-balancing, i.e., redistribute the application's workload among the new set of resources.

Programmable Elasticity. To optimize the performance of new or existing distributed applications while deploying or moving them to the cloud, engineering robust elasticity management components is essential. This is especially vital for applications that do not fit the programming model of (implicit) elastic frameworks like Hadoop, Pig, etc., but require high performance (high throughput and low latency), scalability and elasticity - the best example is the class of datacenter infrastructure applications like key-value stores (e.g., memcached 22, Hyperdex [23]), consensus protocols (e.g., Paxos [15]), distributed lock managers (e.g., Chubby [7]) and message queues. Elasticity frameworks which rely on externally observable resource utilization metrics (CPU, RAM, etc.) are insufficient for such applications (as we demonstrate empirically in Section 5). A distributed key value store, for example, may have high CPU utilization when there is high contention to update a certain set of "hot keys". Relying on CPU utilization to simply add additional compute nodes will only degrade its performance further. Hence, there is an emerging need for a elasticity framework that bridges the gap between implicit and explicit elasticity, allowing the use fine grained application specific metrics (e.g., size of a queue/heap, number of aborted transactions or average number of attempts to acquire certain locks) to build an elasticity management component right into the application without compromising (1) security, by revealing application-level information to the cloud service provider, and (2) portability across different cloud vendors.

Why RMI?. Despite being criticized for introducing direct dependencies across nodes through remote object references, Remote Method Invocation (RMI) and Remote Procedure Call (RPC) remain a popular paradigm [3] for distributed programming, because of their simplicity. Their popularity has led to the development of Apache Thrift [3] with support for RPC across different languages, and the design of cloud computing paradigms like RAMCloud [16] with support for low-latency RPC. However, high-level support for elasticity is limited in existing RPC-like frameworks; and such support is vital to engineering efficient distributed applications and migrating existing applications to the cloud.

Contributions. This paper makes the following technical contributions:

1. ElasticRMI - A framework for elastic distributed objects in Java:

(a) with the same simplicity and ease of use of the Java RMI, handling elasticity at the level of classes and objects, while supporting implicit and explicit elasticity. (Section 2) 


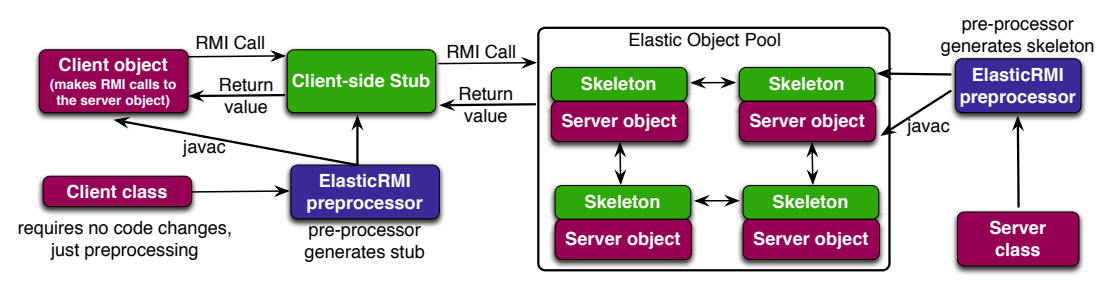

Fig. 1. ElasticRMI - Overview

(b) that enables application developers to dynamically change the number of (server) objects available to handle remote method invocations with respect to the application's workload, without requiring any change to clients (invokers) of remote methods. (Section 3 )

(c) enables flexible elastic scaling by allowing developers to use a combination of resource utilization metrics and fine-grained application-specific information to drive scaling decisions. (Section 3)

2. A runtime system that handles all the low-level mechanics of instantiating elastic objects, monitoring their workload, adding/removing additional objects as necessary and load balancing among them. (Section 4)

3. Performance evaluation of ElasticRMI using elasticity metrics defined by SPEC 24 and using four existing real world applications - Marketcetera financial order processing, Paxos consenus protocol, Hedwig publish/subscribe system and a distributed coordination service. (Section 5)

\section{ElasticRMI - Overview}

Figure1illustrates the architecture of ElAsticRMI. In designing ELASTICRMI, our goals are (1) to retain the simple programming model of Java RMI, and mask the low level details of implementing elasticity like workload monitoring, load balancing, and adding/removing objects from the application developer. (2) require minimal changes, if any, to the clients of an object (3) make ELASTICRMI applications as portable as possible across different IaaS cloud implementations.

\subsection{Elastic Classes and Object Pools}

An ElasticRMI application consists of multiple components (implemented as classes) interacting with each other. The basic elasticity abstraction in ELASTICRMI is an elastic class. An elastic class is also a remote class, and (some) of its methods may be invoked remotely from another JVM. The key difference between an elastic and a regular remote class is that an elastic class is instantiated into a pool of objects (referred to as elastic object pool), with each object executing on a separate JVM. But, the presence of multiple objects in an elastic object pool is transparent to its clients, because the pool behaves as a single remote object. Clients can only interact with the entire object pool, by invoking 
its remote methods. The interaction between a client and an elastic object pool is unicast interaction, similar to Java RMI. The processing of the method invocation, i.e., the method execution happens at a single object in the elastic object pool chosen by the ElasticRMI runtime, and not the client. ElasticRMIs runtime can redirect incoming method invocations to one of the objects in the pool, depending on various factors and performance metrics (Section 4 describes load balancing in detail). The runtime automatically changes the size of the pool depending on the pool's "workload". ELASTICRMI is different from certain frameworks where the same method invocation is multicast and consequently executes on multiple (replicated) objects for fault-tolerance.

\subsection{Shared State and Consistency}

In Java RMI, the state of a remote (server) object is a simple concept, because it resides on a single JVM and there is exactly one copy of all its fields. Clients of the remote object can set the value of instance fields by calling a remote method, and access the values in subsequent method calls. In ELASTICRMI, on the other hand, the entire object pool should appear to the client as a single remote object - thereby necessitating coordination between the objects in the pool to consistently update the values of instance fields. For consistency, we employ an external in-memory key-value store (HyperDex [23] in our implementation) to store the state (i.e., public, private, protected and static fields) of the elastic remote object pool. The key-value store is not used to store local variables in methods/blocks of code and parameters in method declarations. Local variables are instantiated on the JVM in which the object resides. The key-value store is shared between the objects in the pool, and executes on separate JVMs.

\subsection{Stubs and Skeletons}

ELASTICRMI modifies the standard mechanisms used to implement RPCs and Java RMI, as illustrated by Figure 1. The ELAsTICRMI pre-processor analyzes elastic classes to generate stubs and skeletons for client-server communication. As in Java RMI, a stub for a remote object acts as a client's local representative or proxy for the remote object. The caller invokes a method on ELASTICRMI's local stub which (1) initiates a connection with the remote JVM, serializes and marshals parameters, waits for the result of the method invocation and unmarshals the return value/exception before returning it to the sender, and (2) performs load balancing among the objects in the elastic pool as necessary. The stub is generated by the ELASTICRMI preprocessor and is different from the client application, to which the entire object pool appears as a single object, i.e., the existence of a pool of objects is known to the stub but not to the client application. In the remote JVM, each object in the pool has a corresponding skeleton, which in addition to the duties performed in regular Java RMI, can also perform dynamic load balancing based on the CPU utilization of the object and redirect all further method invocations to other objects in the pool after ELASTICRMI decides to shut it down in response to decreasing workload. 


\subsection{Instantiation of Object Pools in a Cluster}

An elastic class can only be instantiated by providing a minimum and maximum number of objects that constitute its elastic object pool. Obviously, instantiating all objects in the pool on separate JVMs on the same physical machine may degrade performance. Hence, ElAsticRMI attempts to instantiate each object in a virtual node in a compute cluster. Virtual nodes can be obtained either (1) from IaaS clouds by provisioning and instantiating virtual machines, or (2) from a cluster management/resource sharing system like Apache Mesos [5. Our implementation of ElasticRMI uses Apache Mesos [5] because it supports both clusters of physical nodes (in private data centers) or virtual nodes (from IaaS clouds). Mesos can also be viewed as a thin resource-sharing layer that manages a cluster of physical nodes/virtual machines. It divides these nodes into "slices" (called resource offers or slave nodes [5]), with each resource offer containing a configurable reservation of $\mathrm{CPU}$ power (e.g., $2 \mathrm{CPUs}$ at $2 \mathrm{GHz}$ ), memory (e.g., 2GB RAM), etc. on one of the nodes being managed. Mesos implements the "slice" abstraction by using Linux Containers (http://lxc.sourceforge.net) to implement lightweight virtualization, process isolation and resource guarantees (e.g., 2CPUs at 2GHz) [5. While instantiating an elastic class, the ElASTICRMI runtime requests Mesos for a specified number of slave nodes, instantiating an object on each slave node.

Mesos aids in portability of ELASTICRMI applications, just like the JVM aids the portability of Java applications. Mesos can be installed on private data centers and many public cloud offerings (like Google Compute Engine, Amazon EC2, etc.). As long as Mesos is available, ElasticRMI applications can be executed, making them portable across different cloud vendors.

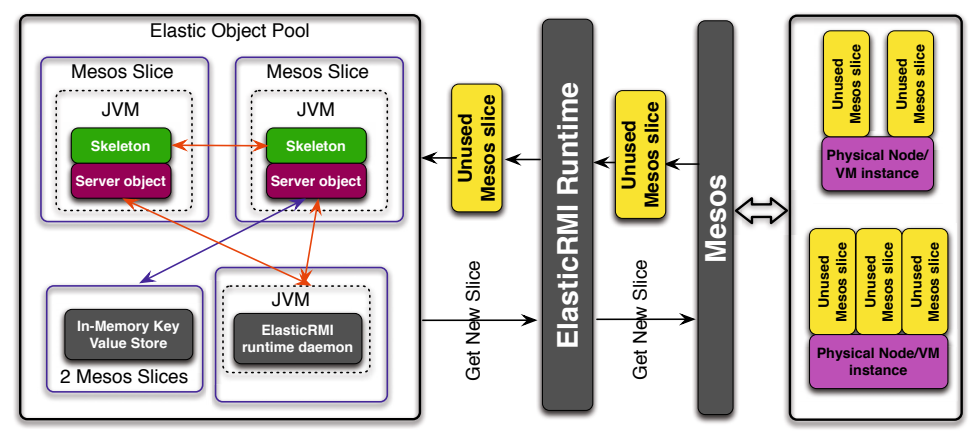

Fig. 2. ElasticRMI - Server Side

\subsection{Automatic Elastic Scaling}

The key objective of ELASTICRMI is to change the number of objects in the elastic pool based on its workload. The "workload" of an elastic object can have several application-specific definitions, and consequently, ELASTICRMI allows 
programmers to define the workload of an elastic class and specify the conditions under which objects should be added or removed from the elastic pool. This can be done by overriding select methods in the ELASTICRMI framework, as discussed in detail in (the next) Section 3.1. During the lifetime of the elastic object, the ELASTICRMI runtime monitors a elastic object pool's workload and decides whether to change its size either based on default heuristics or by applying the programmer's logic by invoking the overridden methods discussed above.

If a decision has been made to increase the size of the pool, the ELASTICRMI runtime interacts with the Mesos master node to request additional compute resources. If the request is granted, ELASTICRMI runtime instantiates the additional object, and adds it to the pool (See Figure 2 for an illustration). If the decision is to remove an object, ELASTICRMI communicates with its skeleton to redirect subsequent remote method calls to other objects in the pool. Once redirection starts, ELASTICRMI sends a SHUTDOWN message to the object. The object acknowledges the message, and waits for all pending remote method invocations to finish execution or throw exceptions indicating abnormal termination. Then the object notifies the ELASTICRMI runtime that it is ready to be shutdown. ElasticRMI terminates the object and relinquishes its slice to Mesos. This slice is then available to other elastic objects in the cluster, or for subsequent use by the same elastic object if a decision is made in the future to increase the size of its pool.

\section{Programming with ElasticRMI}

This section illustrates the use of ELASTICRMI for both implicit and explicit elasticity through examples, along with an overview of how to make such decisions with a global view of the entire application. Our implementation also includes a preprocessor similar to rmic which in addition to generating stubs and skeletons, converts ELASTICRMI programs into plain Java programs that can be compiled with the javac compiler.

\subsection{ElasticRMI Class Hierarchy}

A distributed application built using ELASTICRMI consists of interfaces declaring methods and classes implementing them. The key features of ELASTICRMI API (Figure 3) are:

- java.elasticrmi is the top-level package for programming ELASTICRMI server classes.

- An elastic interface is one that declares the set of methods that can be invoked from a remote JVM (client). All elastic interfaces must extend ELASTICRMI's marker interface - java.elasticrmi.Elastic, either directly or indirectly.

- The Elasticobject class implements all the basic functionalities of ELASTICRMI. An application-defined class becomes elastic by implementing one or more elastic interfaces and by extending ElasticObject. 


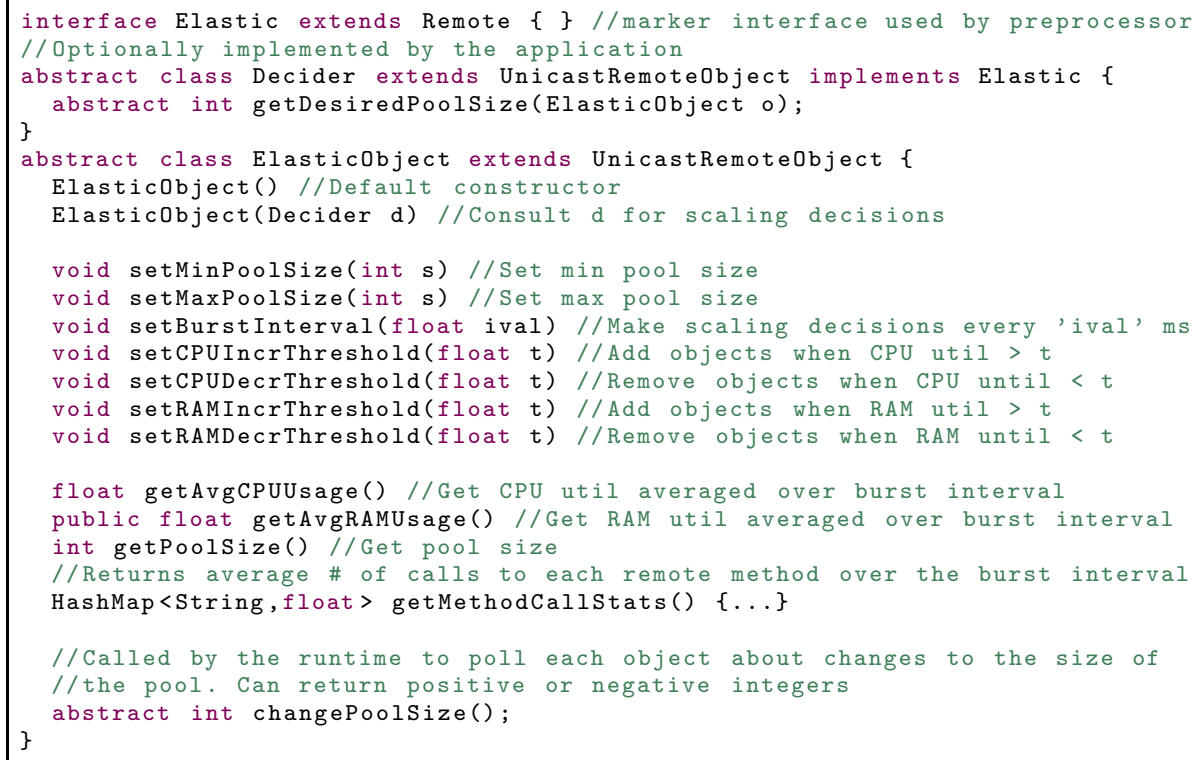

Fig. 3. A snapshot of the ElasticRMI server-side API.

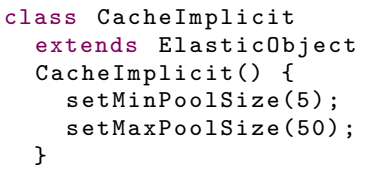

(a) Implicit elasticity

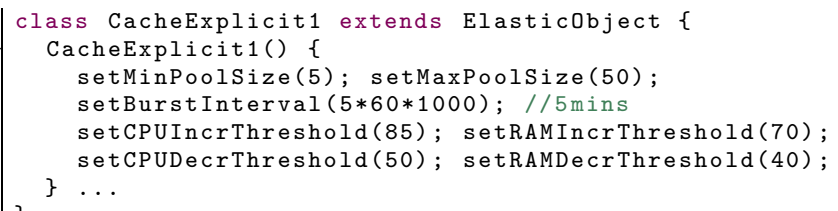
...

(b) Explicit elasticity using coarse-grained metrics.

Fig. 4. Example of two distributed cache classes implemented in ElasticRMI

\subsection{Programming with Implicit Elasticity}

ELASTICRMI supports implicit elastic scaling, using average CPU utilization across the objects in an elastic pool as the default coarse-grained metric. A time interval, referred to as the burst interval (default 60s) is used to decide whether to change the size of elastic object pool. ELASTICRMI measures the average CPU utilization of each object in the elastic pool every 60s - objects are added (in increments of 1 object) when the average utilization exceeds $90 \%$ and removed when average utilization falls below 60\%. Figure 4 a shows an example of a distributed cache class (e.g., a web cache, content/object cache) that relies on ELAsticRMI's implicit elasticity mechanisms. The programmer simply implements a cache store based on some well-known algorithm and specifying the minimum and maximum size of the pool, without worrying about adapting to new resources and load balancing. 


\subsection{Programming with Explicit Elasticity}

ELASTICRMI also allows programmers to explicitly define the workload of an elastic class and specify the conditions under which objects should be added or removed from the elastic pool. Workload definitions can either be coarse-grained or fine-grained.

Coarse-Grained Metrics. A programmer can override the default burst interval, and the average CPU utilization thresholds used for changing the number of objects in the pool by calling the appropriate methods (setBurstInterval(...), setCPUIncrThreshold(...) and setCPUDecrThreshold(...)) in java.elasticrmi.Elasticobject which is available in all elastic classes since they extend Elasticobject (see Figure 3).

Figure 4b shows an example of a cache class that changes the CPU and memory (RAM) utilization thresholds that trigger elastic scaling. The core ELASTICRMI API includes specific methods to set CPU and memory thresholds because they are commonly used for elastic scaling - if both CPU and RAM thresholds are set, the runtime interprets them using a logical OR, i.e., in the example shown in Figure $4 \mathrm{~b}$ the ELASTICRMI runtime increases the size of the pool by in increments of 1 object every five minutes, either if average CPU utilization exceeds $85 \%$ or if average memory utilization exceeds $70 \%$ across the JVMs in the elastic object pool.

Fine-grained Metrics. ElasticRMI provides additional support, through the changePoolsize method (see Figure 3) which can be overridden by any elastic class. The runtime periodically (every "burst interval") invokes changePoolsize to poll each object in the elastic object pool, about desired changes to the size of the pool. The method returns an integer - positive or negative corresponding to increasing or decreasing the pool's size. The values returned by the various objects in the pool are averaged to determine the number of objects that have to be added/removed. The logic used to decide on elastic scaling is left to the developer, and it may be based on (1) parameters of the JVM on which each object resides, (2) properties of shared instance fields of the elastic object, or of data structures used by the object, e.g., number of pending client operations stored in a queue, and (3) metrics computed by the object like average response time, throughput, etc. ELASTICRMI allows classes to use only a single decision mechanism for elastic scaling, i.e., if changePoolsize is overridden, then scaling based on CPU/Memory utilization is disabled.

Figure 5 illustrates the use of changePoolsize to make scaling decisions. The CacheExplicit2 class is implemented to use metrics specific to distributed object caches, e.g., avgLockAcqFailure (which measures the failure rate of acquiring write locks to ensure consistency during a put operation on the cache) and avgLockAcqLatency (which measures the average latency to acquire write locks) to make decisions about changing the size of the elastic object pool. In Figure 5, the CacheExpiicit2 class does not add new objects to the pool when there is a lot of contention. When the failure rate for acquiring write locks (avgLockAcqFailure is greater than $50 \%$ ) or 


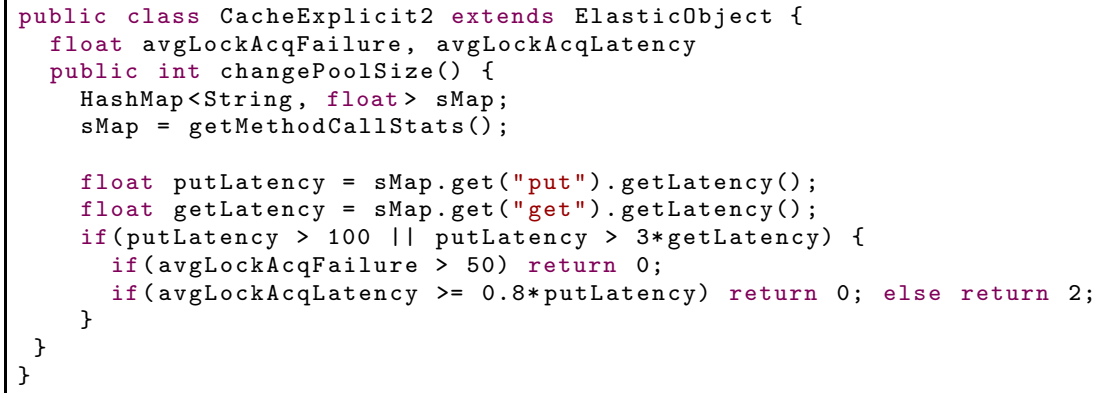

Fig. 5. A distributed cache class which relies on ELAsticRMI's explicit elasticity support using fine-grained application-specific metrics.

when the predominant component of putLatency is avgLockAcqLatency, no additional objects are added to the pool because there is already high contention among objects serving client requests to acquire write locks. When these conditions are false, the size of the pool is increased by two - controlling the number of objects added is another feature of changePoolsize.

Making Application-Level Scaling Decisions. The mechanisms described above involve makes scaling decisions local to an elastic class, and may not be optimal for applications using multiple elastic classes (where the application contains tiers of elastic pools). ELASTICRMI also supports decision making at the level of the application using the Decider class. It is the developer's responsibility to ensure that elastic objects being monitored communicate with the monitoring components, either by using remote method invocations or through message passing. The ELASTICRMI runtime assumes responsibility for calling changePoolsize method of the monitoring class to get the desired size of each elastic object pool, and determines whether objects have to be added or removed. Due to space limitations, we refer the reader to our tech report for additional details [20].

\section{The ElasticRMI Runtime}

The runtime (1) handles shared state among the objects in an elastic object pool, (2) instantiates each object of a pool, (3) performs load balancing, and (4) is responsible for fault-tolerance.

\subsection{Shared State and Consistency}

The objects in the elastic object pool coordinate to update the state of its instance fields (public, private, protected) and static fields. For consistency, we use HyperDex 23, a distributed in-memory key-value store (with strong consistency). Using an in-memory store provides the same data durability guarantees as Java RMI which stores the state of instance fields in RAM in a single Java 
virtual machine heap. HyperDex is different from the distributed cache in the examples of Section 3, which is used for illustration purposes only. The ElastiCRMI preprocessor translates reads and writes of instance and static fields into get $(. .$.$) and put (. .$.$) method calls of HyperDex.$

Figure [6] shows a simple elastic class C1 and how it is transformed by the ELASTICRMI preprocessor to insert calls to HyperDex (abstracted by store ) and ElasticRMI's runtime (abstracted by ERMI). For variable $\mathrm{x}, \quad \mathrm{c} 1 \$ \mathrm{x}$ is used as the key in store.

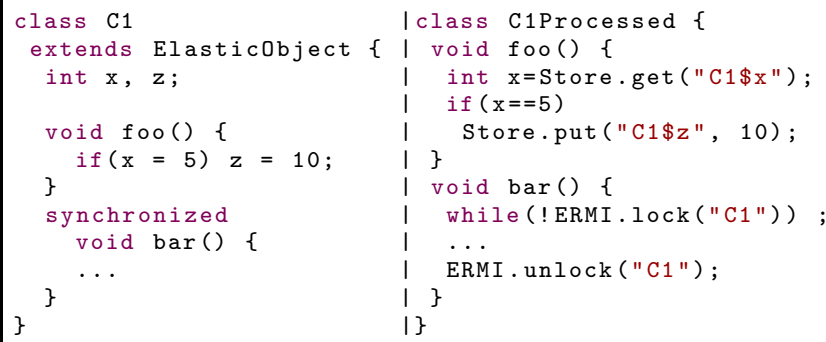

Fig. 6. Handling shared state through an in-memory store (HyperDex 23, here)

For synchronized meth-

ods, ElASTicRMI uses a lock per class named after the class - the example in Figure 6 uses a lock called "C1".

If an elastic class has an instance or static field $f$, a method call on $f$ is handled as follows:

- If $f$ is a remote or elastic object, the method invocation is simply serialized and dispatched to the remote object or pool as the case may be.

- Else, if $m$ is synchronized. the method call is handled as in Figure 6, but the runtime acquires a lock on $f$ through HyperDex. In this case, ElAsticRMI guarantees mutual exclusion for the execution of $f . m(\ldots)$ with respect to other methods of $f$.

- Else, (i.e., $m$ is neither remote, elastic nor synchronized), f.m(...) involves retrieving $f$ from HyperDex and executing $m(\ldots)$ locally on an object in the elastic object pool, and storing $f$ back into HyperDex after $m(\ldots)$ has completed executing.

ELASTICRMI aims to increase parallelism and hence the number of remote method executions per second when there is limited or no shared state. Increasing shared state increases latency due to the network delays involved in accessing HyperDex. Having shared state and mutual exclusion through locks or synchronized methods further decreases parallelism. However, we note that this is not a consequence of ELASTICRMI, but rather dependent on the needs of the distributed application. If the developer manually implements all aspects of elasticity by using plan Java RMI and an existing tool like Amazon CloudWatch+Autoscaling, he still has to use something like a key-value store to handle shared state. ElASTICRMI cannot and does not attempt to eliminate this problem - it is up to the programmer to reduce shared state. 
Note that ELASTICRMI does not guarantee a transactional (ACID) execution of $m(\ldots)$ with respect to other objects in the pool, and using synchronized does not provide ACID guarantees either in RMI or in ELASTICRMI.

\subsection{Instantiation of Elastic Objects}

An elastic class can only be instantiated by providing a minimum $(\geq 2)$ and maximum number of objects that constitute its elastic object pool (see Figure 3). During instantiation, if the minimum number of objects is $k$, ElasticRMI's runtime creates $k$ objects on $k$ new JVM instances on $k$ virtual nodes (Mesos slices), if $k$ virtual nodes are available from Mesos [5]. If only $l<k$ are available, then only $l$ objects are created. Under no circumstance does ELASTICRMI create two or more JVM instances on the same slice obtained from Mesos. Then, ELAstICRMI instantiates the HyperDex on one additional Mesos slice, and continues to monitor the performance of the HyperDex over the lifetime of the elastic object. ELASTICRMI may add additional nodes to HyperDex as necessary. ELASTICRMI also enables administrators to be notified if the utilization of the Mesos cluster exceeds or falls below (configurable) thresholds, enabling the proactive addition of computing resources before the cluster runs out of slices.

\subsection{Load Balancing}

Unlike websites or web services, where load balancing has to be performed on the server-side, ELASTICRMI has the advantage that both client and server programs are pre-processed to generate stubs and skeletons respectively. Hence, we employ a hybrid load balancing model involving both stubs and skeletons - note that all load balancing code is generated by the pre-processor, and the programmer does not have to handle any aspect of it explicitly. Please also note that this section describes the simple load balancing techniques used in ELASTICRMI, but we do not claim to have invented a new load balancing algorithm.

On the server side, the runtime, while instantiating skeletons in an elastic object pool, assigns monotonically increasing unique identifiers (uid) to each skeleton, and stores this information in HyperDex. The skeleton with the lowest uid is chosen by the runtime to be the leader of the elastic object pool, called the sentinel. This is similar to leader election algorithms that use a so-called "royal hierarchy" among processes in a distributed system. The sentinel, in addition to performing all the regular functions (forwarding remote method invocations) to its object in the pool, also helps in load balancing. The client stub created by the ELASTICRMI preprocessor (see Section 2.4) has the ability to communicate with the sentinel to invoke remote methods. While contacting the sentinel for the first time, the stub on the client JVM requests the identities (IP address and port number) of the other skeletons in the pool from the sentinel.

For load-balancing on the client-side, the stub then re-directs subsequent method invocations to other objects in the object pool either randomly or in a round-robin fashion. If an object has been removed from the pool after its 
identity is sent to a stub, i.e., if the sending itself fails, the remote method invocation throws an exception which is intercepted by the client stub. The stub then retries the invocation on other objects including the sentinel. If all attempts to communicate with the elastic object pool fail, the exception is propagated to the client application.

For load-balancing on the server side, the sentinel is also responsible for collecting and periodically broadcasting the state of the pool - number of objects, their identities and the number of pending invocations - to the skeletons of all its members. We use the JGroups group communication system for broadcasts. If the sentinel notices that any skeleton is overloaded with respect to others, it instructs the skeleton to redirect a portion of invocations to a set of other skeletons. To decide the number of invocations that have to be redirected from each overloaded skeleton, our implementation of the sentinel uses the first-fit greedy bin-packing approximation algorithm (See http://en.wikipedia.org/wiki/Bin_packing_problem). As mentioned in the previous paragraph, client-side load balancing occurs at the stub while server-side load balancing involves skeletons and the sentinel which monitor the state of the JVM and that of the elastic object pool to redirect incoming method invocations.

\subsection{Fault Tolerance}

Existing RMI applications implement fault tolerance protocols on top of Java RMI's fault- and fault tolerance model, where objects typically reside in main memory, and can crash in the middle of a remote method invocation. !e want to preserve it to make adoption of ELAsTICRMI easier. In short, ELASTICRMI does not hide/attempt to recover from failures of client objects, key-value store (HyperDex) or the server-side runtime processes and propagates corresponding exceptions to the application. However, ELASTICRMI attempts to recover from failures of the sentinel and from Mesos-related failures. Sentinel failure triggers the leader election algorithm described in 4.3 to elect a new sentinel, and mesosrelated failures affect the addition/removal of new objects until Mesos recovers.

\section{Evaluation}

In this section, we evaluate the performance of ELASTICRMI, using metrics relevant to elasticity. Due to space limitations, we refer the reader to our tech report for additional details 20 .

\subsection{Elasticity Metrics}

Measuring elasticity is different from measuring scalability. (Recall that) Scalability is the ability of a distributed application to increase its "performance" proportionally (ideally linearly) with respect to the number of available resources, while elasticity is the ability of the application to adapt to increasing or decreasing workload; adding or removing resources to maintain a specific level of 
"performance" or "quality of service (QoS)" 24]. Performance/QoS is specific to the application - typically a combination of throughput and latency. A highly elastic system can scale to include newer compute nodes, as well as quickly provision those nodes. There are no standard benchmarks for elasticity, but the Standard Performance Evaluation Corporation (SPEC) has recommended elasticity metrics for IaaS and PaaS clouds 24].

Agility. This metric characterizes the ability of a system provisioned to be as close to the needs of the workload as possible [24. Assuming a time interval $\left[t, t^{\prime}\right]$, which is divided into $N$ sub-intervals, Agility maintained over $\left[t, t^{\prime}\right]$ can be defined as:

$$
\frac{1}{N}\left(\sum_{i=0}^{N} \operatorname{Excess}(i)+\sum_{i=0}^{N} \operatorname{Shortage}(i)\right)
$$

where (1)Excess $(i)$ is the excess capacity for interval $i$ as determined by Cap_prov $(i)$ - Req_min $(i)$, when Cap_prov $(i)>$ Req_min $(i)$ and zero otherwise. (2) Shortage $(i)$ is the shortage capacity for interval $i$ as determined by Req_min $(i)-C a p \_p r o v(i)$, when Cap_prov $(i)<R e q \_m i n(i)$ and zero otherwise. (3) Req_min $(i)$ is the minimum capacity needed to meet an application's quality of service (QoS) at a given workload level for an interval $i$. (4) Cap_prov $(i)$ is the recorded capacity provisioned for interval $i$, and (5) $N$ is the total number of data samples collected over a measurement period $\left[t, t^{\prime}\right]$, i.e., one sample of both Excess $(i)$ and Shortage $(i)$ is collected per sub-interval of $\left[t, t^{\prime}\right]$.

Elasticity measures the shortage and excess of computing resources over a time period. For example, a value of elasticity of 2 over $\left[t, t^{\prime}\right]$ when there is no excess means that there is a mean shortage of 2 "compute nodes" over $\left[t, t^{\prime}\right]$. For an ideal system, agility should be as close to zero as possible - meaning that there is neither a shortage nor excess. Agility is a measurement of the ability to scale up and down while maintaining a specified QoS. The above definition of agility will not be valid in a context where the QoS is not met. It should be noted that there is ongoing debate over whether Shortage and Excess should be given equal weightage 24] in the Agility metric, but there are disagreements over what the weights should be otherwise.

Provisioning Interval. Provisioning Interval is defined as the time needed to bring up or drop a resource. This is the time between initiating the request to bring up a new resource, and when the resource serves the first request.

\subsection{ElasticRMI Applications for Evaluation and Workloads}

We have re-implemented four existing applications using ELASTICRMI to add elasticity management components to them. This does not involve altering the percentage of shared state or the frequency of accesses (reads or writes) to said state. 


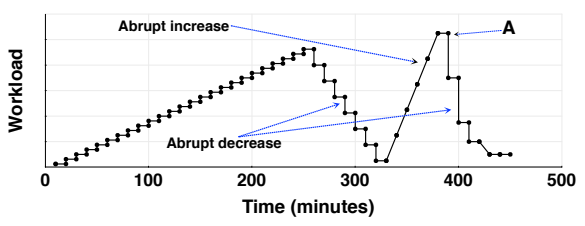

(a) Pattern for abruptly changing workload for all four systems. The pattern remains the same, but the meaning and magnitude vary for the four systems.

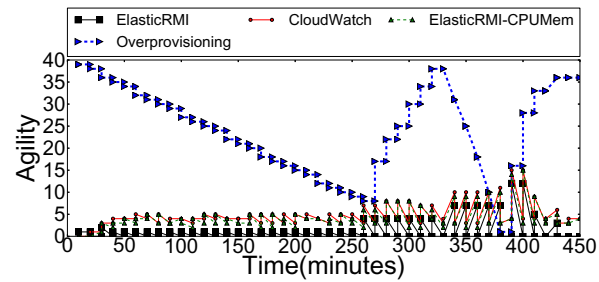

(c) Marketcetera - abrupt workload.

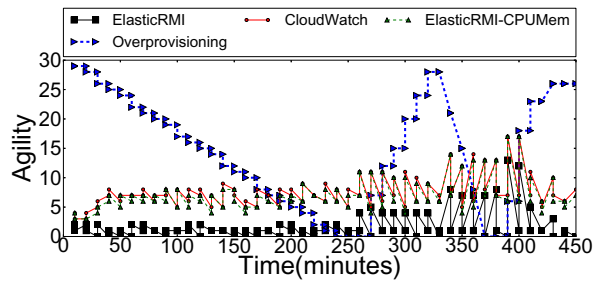

(e) Hedwig - abrupt workload.

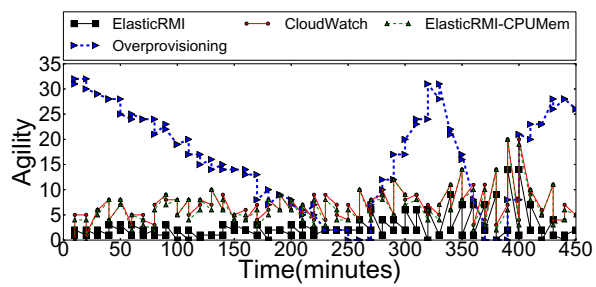

(g) Paxos - abrupt workload.

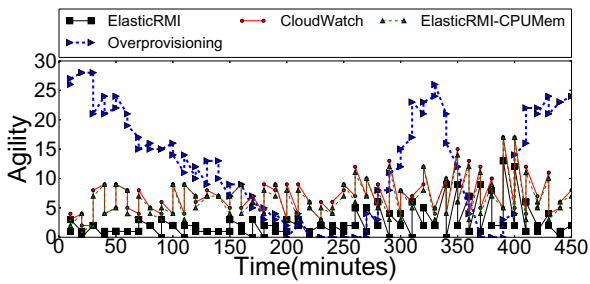

(i) DCS - abrupt workload.

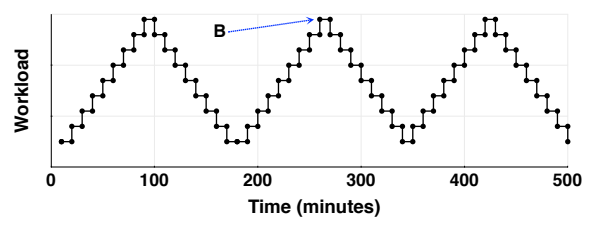

(b) Cyclical workload example for all four systems. As in Figure 7a, the pattern remains the same for all four systems but the meaning and magnitude are different.

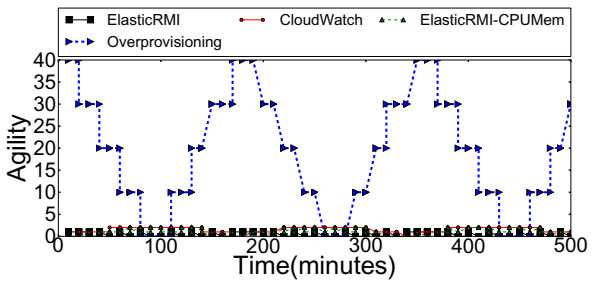

(d) Marketcetera - cyclical workload.

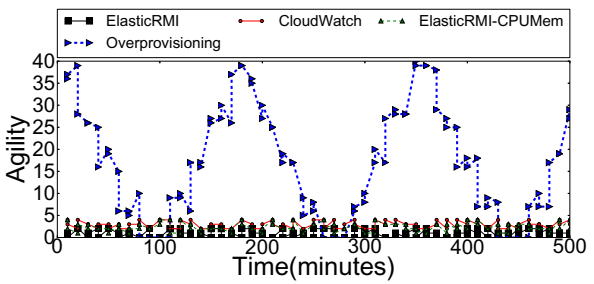

(f) Hedwig - cyclical workload.

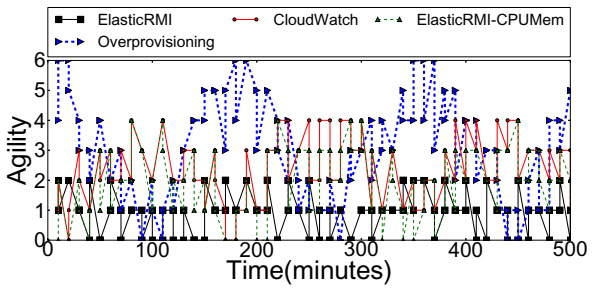

(h) Paxos - cyclical workload.

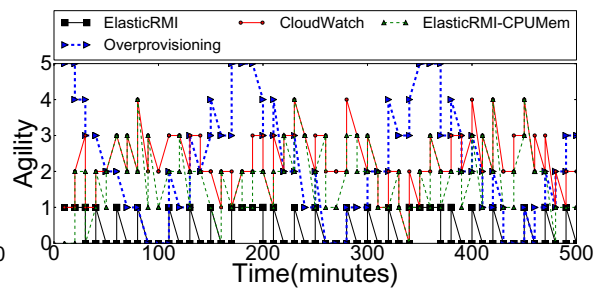

(j) DCS - cyclical workload.

Fig. 7. Elasticity Benchmarking of Marketcetera order routing, Hedwig, Paxos and DCS. We compare the ELASTICRMI implementation of these applications with two other systems described in Section 5.4 . 
Marketcetera [11] Order Routing. Marketcetera is an NYSE-recommended algorithmic trading platform. The order routing system is the component that accepts orders from traders/automated strategy engines and routes them to various markets (stock/commodity), brokers and other financial intermediaries. For fault-tolerance, the order is persisted (stored) on two nodes. The workload for this system is a set of trading orders generated by the simulator included in the community edition of Marketcetera [11.

Apache Hedwig [14]. Hedwig is a topic-based publish-subscribe system designed for reliable and guaranteed at-most once delivery of messages from publishers to subscribers. Clients are associated with (publish to and subscribe from) a Hedwig instance (also referred to as a region), which consists of a number of servers called hubs. The hubs partition the topic ownership among themselves, and all publishes and subscribes to a topic must be done to its owning hub. The workload for this system is a set of messages generated by the default Hedwig benchmark included in the implementation.

Paxos [15]. Paxos is a family of protocols for solving consensus in a distributed system of unreliable processes. Consensus protocols are the basis for the state machine approach to distributed computing, and for our experiments we implement Paxos using a widely-used specification by Kirsch and Amir [15. The workload for this system is the default benchmark included in libpaxos [21].

$D C S$. DCS is a distributed co-ordination service for datacenter applications, similar to Chubby 7 and Apache Zookeeper [25]. DCS has a hierarchical name space which can be used for distributed configuration and synchronization. Updates are totally ordered. The workload for this system is the default benchmark included in Apache Zookeeper [25].

\subsection{Workload Pattern}

To measure how well the system adapts to the changing workload, we use two patterns shown in Figures $7 \mathrm{a}$ and $7 \mathrm{~b}$. These two patterns capture all common scenarios in elastic scaling which we have observed by analyzing real world applications. The abrupt pattern shown in Figure 7 a has all possible scenarios regarding abrupt changes in workload - gradual non-cyclic increase, gradual decrease, rapid increases and rapid decrease in workload. A cyclic change in workload is shown by the second pattern in Figure $7 \mathrm{~b}$, So, together the patterns in Figures $7 \mathrm{a}$ and $7 \mathrm{~b}$ exhaustively cover all elastic scaling scenarios we observed. Note however, that although the pattern remains the same for varying the workload while evaluating all the four systems, the magnitude differs depending on the benchmark used, i.e., the values of points $\mathrm{A}$ and B in Figures $7 \mathrm{a}$ and $7 \mathrm{~b}$ are different for the four systems depending on the benchmark. Point A, for example, is 50,000 orders/s for Marketcetera, 75,000 updates/s for DCS, 24,000 consensus rounds/s for Paxos and 30,000 messages/s for Hedwig. We set Point B at 20\% above Point A - note that the specific values of Points A and B are immaterial because we are only measuring adaptability and not peak performance. 


\subsection{Overprovisioning and CloudWatch}

We compare the ELASTICRMI implementation of the applications in Section 5.2 with the existing implementations of the same applications in two deployment scenarios - (1) Overprovisioning and (2) Amazon AutoScaling + CloudWatch [4] [1. The overprovisioning deployment scenario is similar to an "oracle" - the peak workload arrival rate i.e., point A for the abruptly changing workload and point B for the cyclic workload are known a priori to the oracle; and the number of nodes required to meet a desired QoS (throughput, latency) at A and B respectively is determined by the oracle through experimental evaluation. The oracle then provisions the application on a fixed set of nodes - the size of which is enough to maintain the desired QoS even at the peak workload arrival rate (A and B respectively). In a nutshell, the over provisioning scenario can be described as "knowing future workload patterns and provisioning enough resources to meet its demands". Overprovisioning is the alternative to elastic scaling - there are going to be excess provisioned resources when the workload is below the peak ( $\mathrm{A}$ and $\mathrm{B}$ ), but provisioning latency is zero because all necessary resources are always provisioned. In the CloudWatch scenario, we use a monitoring service - Amazon CloudWatch to collect utilization metrics (CPU/Memory) from the nodes in the cluster and use conditions on these metrics to decide whether to increase or decrease the number of nodes. The ELASTICRMI implementation of the above applications, however, uses a combination of resource utilization and application-level properties specific to Marketcetera, DCS, Paxos and Hedwig respectively to decide on elastic scaling. Since ElasticRMI and CloudWatch are two different systems, we also compare the ELASTICRMI implementation of the four applications with another version, referred to as ElasticRMI-CPUMem in Figure[7, where no application-level properties are used but only the the conditions based on CPU/Memory utilization in CloudWatch are used.
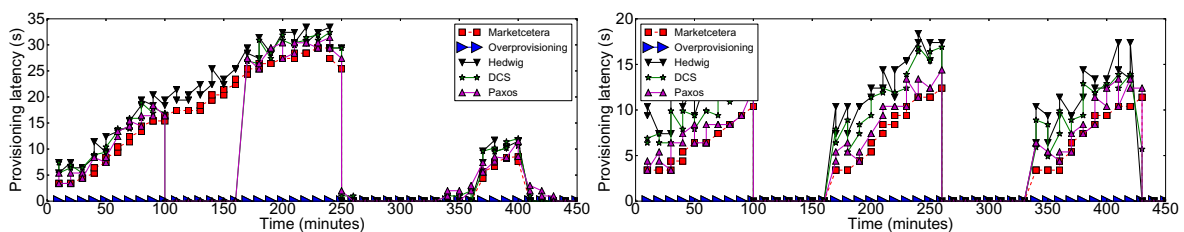

(a) Provisioning latency - Abrupt Work-(b) Provisioning latency - Cyclic Workload load

Fig. 8. Provisioning latency in seconds for ElasticRMI and Overprovisioning (which is always 0). Provisioning latency for Amazon CloudWatch is not plotted because it is in several minutes and hence well above that of both ElasticRMI and Overprovisioning. You can see repeating patterns corresponding to the cyclic workload.

\subsection{Agility Results}

In this section, we compare the Agility of the ElasticRMI implementation of all four systems against Overprovisioning, CloudWatch and ElasticRMI-CPUMem. 
Marketcetera Order Routing. The relevant QoS metrics for the order routing subsystem are order routing throughput, which is the number of orders routed from traders to brokers/exchanges per second and order propagation latency, which is the time taken for an order to propagate from the sender to the receiver. We compare the elasticity of the three deployments of the order routing system described in Section 5.4 The results are illustrated in Figure 7 Figures $7 \mathrm{c}$ and $7 \mathrm{~d}$ plot the agility over the same time period as in Figures $7 \mathrm{a}$ and $7 \mathrm{~b}$ for all the four deployments. From Figures $7 \mathrm{c}$ and $7 \mathrm{~d}$, we observe that the agility of ELASTICRMI is better than CloudWatch, ElasticRMI-CPUMem and overprovisioning. Ideally, agility must be zero, because agility is essentially a combination of resource wastage or resource under-provisioning. We observe that for abruptly changing workloads, agility of ELASTICRMI is close to 1 most of the time, and increases to 5 during abrupt changes in workload. We also observe that the the agility of ELASTICRMI oscillates between 0 and a positive value frequently. This proves that the elastic scaling mechanisms of ELASTICRMI perform well in trying to achieve optimal resource utilization, i.e., react aggressively by trying to push agility to zero. In summary, the average agility of ELASTICRMI for abruptly changing workload is 1.37. As expected, the agility of overprovisioning is the worst, up to $24 \times$ that of ELASTICRMI. This is not surprising, because its agility does reach zero at peak workload, when the agility of ELASTICRMI is 5, thus illustrating that overprovisioning optimizes for peak workloads. The average agility of overprovisioning is 17.2 for the cyclical workload and 24.1 for abruptly changing workload. CloudWatch performs much better than overprovisioning, but it is less agile than ELASTICRMI. Its agility is approximately $3.4 \times$ that of ELASTICRMI on average for abruptly changing workloads, and it does not oscillate to zero frequently like ELASTICRMI. The agility of ElasticRMI-CPUMem is approximately equal to CloudWatch and is $3.02 \times$ that of ELAsTiCRMI on average - this is in spite of ElasticRMI-CPUMem and CloudWatch being different systems. having different provisioning latencies. This is because the same conditions are used to decide on elastic scaling and because the provisioning latency of CloudWatch is well within the sampling interval of 10 minutes used in Figure 7

Figure $7 \mathrm{~d}$ shows that the agility of ELASTICRMI is again better than that of CloudWatch and overprovisioning for cyclic workloads. We also observe that as in the case of abrupt workloads, the agility of ELASTICRMI tends to decrease to zero more frequently than the other two deployments. Figure $7 \mathrm{~d}$ also demonstrates the oscillating pattern in the agility of overprovisioning - the initial agility is high (and comes from Excess), and as the workload increases, Excess decreases, thereby decreasing Agility and bringing it to zero corresponding to Point B. Then Excess increases again as the workload decreases thereby increasing Agility. This repeats three times. As expected, the agility of ElasticRMICPUMem is similar to that of CloudWatch.

Hedwig. The relevant QoS metrics for Hedwig are also throughput and latency - the number of messages published per second and time taken for the message to propagate from the publisher to the subscriber. Figures $7 \mathrm{e}$ and $7 \mathrm{f}$ illustrate 
the agility corresponding to our experiments with Hedwig. From Figures 7 e and 7f. we observe similar trends as in the case of Marketcetera order processing. ELASTICRMI has lower agility values than the other two deployments, and the agility of ElasticRMI tends to oscillate between zero and a positive value. The agility values of CloudWatch are more than $4.5 \times$ that of ELASTICRMI, on average for abrupt workloads and $3 \times$ that of ELASTICRMI for cyclic workloads. As expected, the agility of over provisioning is the highest, and is worse than the values observed for Marketcetera in the case of cyclic workloads. We also observe a similar oscillating trend in the agility values of the overprovisioning deployment as in Marketcetera, but the agility values oscillate more frequently because Req_min $(i)$ - the minimum capacity needed to maintain QoS under a certain workload changes more erratically than Marketcetera due to the replication and at-most once guarantees provided by Hedwig for delivered messages.

Paxos. The relevant QoS metrics for Paxos are the number of consensus rounds executed successfully per second, and the time taken to execute a consensus round. Figures $7 \mathrm{~g}$ and $7 \mathrm{~h}$ illustrate the agility corresponding to our experiments with Paxos. From Figures $7 \mathrm{~g}$ and $7 \mathrm{~h}$, we observe similar trends to Hedwig and Marketcetera. The agility of CloudWatch in this case is $6.6 \times$ than of ElASTICRMI, on average for abrupt workloads and $2.2 \times$ that of ElAsticRMI for cyclic workloads. We also observe that the agility of ELASTICRMI returns to zero (the ideal agility) most frequently among the three deployments.

$D C S$ The relevant QoS metrics for DCS are the number of updates to the hierarchical name-space per second and the end-to-end latency to perform an update as measured from the client. Figures $7 \mathrm{i}$ and $7 \mathrm{j}$ illustrate the agility corresponding to our experiments with DCS. From Figures 7i and 7j, we observe that the agility of CloudWatch in this case is $7.2 \times$ than of ELASTICRMI, on average for abrupt workloads and $3.2 \times$ that of ELASTICRMI for cyclic workloads.

\subsection{Provisioning Latency}

Figures $8 \mathrm{a}$ and $8 \mathrm{~b}$ plot the provisioning latency of ELASTICRMIfor both abrupt and cyclic workloads. We observe that the provisioning latency of ElAsticRMI is less than 30 seconds in all cases, which compares very favorably to the time needed to provision new VM instances in Amazon CloudWatch (which is in the order of several minutes, and hence omitted from Figure 8). Provisioning latency is zero for the overprovisioning scenario, and that is the main purpose of overprovisioning - to have resources always ready and available. Also, we observe that as the workload increases, provisioning interval also increases, due to the overhead in determining the remote method calls that have to be redirected and also due to increasing demands on the resources of the sentinel object in ELASTICRMI's object pools. 


\section{Related Work}

J-Orchestra 89 automatically partitions Java applications and makes them into distributed applications running on distinct JVMs, by using byte code transformations to change local method calls into distributed method calls as necessary. The key distinctions between J-Orchestra and ElAsTicRMI are that (1) J-Orchestra tackles the complex problem of automatic distribution of Java programs while ELASTICRMI aims to add elasticity to already distributed programs and (2) ElASTICRMI partitions different invocations of a single remote method.

Self-Replicating Objects (SROs) [17] is a new elastic concurrent programming abstraction. An SRO is similar to an ordinary .NET object exposing an arbitrary API but exploits multicore CPUs by automatically partitioning its state into a set of replicas that can handle method calls (local and remote) in parallel, and merging replicas before processing calls that cannot execute in replicated state. SRO also does not require developers to explicitly protect access to shared data; the runtime makes all the decisions on synchronization, scheduling and splitting/merging state. Live Distributed Objects (LDO) [19] is a new programming paradigm and a platform, in which instances of distributed protocols are modeled as live distributed objects. Live objects can be used to represent distributed multi-party protocols and application components. Shared-state and synchronization between the objects is maintained using Quicksilver [18, a group communication system. Automatic scaling is not supported and must be explicitly implemented by the programmer using the abstractions provided by LDO.

Quality Objects $(\mathrm{QuO})[$ [ is a seminal framework for providing quality of service (QoS) in network-centric distributed applications. When the requirements are not being met, $\mathrm{QuO}$ provides the ability to adapt at many levels in the system, including the middleware layer responsible for message transmission. In contrast to QuO, ElASTICRMI attempts to increase quality of service by changing the size of the remote object pool, and does not change the protocols used to transmit remote method invocations.

\section{Conclusions}

We have described the design and implementation of ELASTICRMI and have demonstrated through empirical evaluation using real-world applications that it is effective in engineering elastic distributed applications. Our empirical evaluation also demonstrates that relying solely on externally observable metrics like CPU/RAM/network utilization decreases elasticity, as demonstrated by the high agility values of CloudWatch. We have shown that our implementation of ELASTICRMI reduces resource wastage, and is sufficiently agile to meet the demands of applications with dynamically varying workloads. Through an implementation using Apache Mesos, we ensure portability of ELASTICRMI applications across Mesos installations, whether it is a private datacenter or a public cloud or a hybrid deployment between private data centers and public clouds. 
We have demonstrated that ELASTICRMI applications can use fine-grained application specific metrics without revealing those metrics to the cloud infrastructure provider, unlike CloudWatch.

\section{References}

1. Amazon Web Services (AWS) Inc. Amazon CloudWatch (2012), http://aws . amazon.com/cloudwatch/

2. Apache Pig (2013), http://pig.apache.org

3. Apache Thrift (2012), http://thrift.apache.org/

4. AWS Inc. Amazon Auto Scaling (2012), http://aws.amazon.com/autoscaling/

5. Hindman, B., Konwinski, A., Zaharia, M., Ghodsi, A., Joseph, A., Katz, R., Shenker, S., Stoica, I.: Mesos: A Platform for Fine-grained Resource Sharing in the Data Center. In: NSDI 2011 (2011), http://incubator.apache.org/mesos/

6. BBN Technologies. Quality Objects (QuO) (2006), http://quo.bbn.com/

7. Burrows, M.: The Chubby Lock Service for Loosely-coupled Distributed Systems. In: OSDI 2006 (2006)

8. Tilevich, E., Smaragdakis, Y.: J-Orchestra: Automatic Java Application Partitioning. In: Magnusson, B. (ed.) ECOOP 2002. LNCS, vol. 2374, pp. 178-204. Springer, Heidelberg (2002)

9. Tilevich, E., Smaragdakis, Y.: Portable and Efficient Distributed Threads for Java. In: Jacobsen, H.-A. (ed.) Middleware 2004. LNCS, vol. 3231, pp. 478-492. Springer, Heidelberg (2004)

10. Elastic Map Reduce (2013), http://aws.amazon.com/elasticmapreduce/

11. Miller, G., Kuznets, T., Agostino, R.: Marketcetera Automated Trading Platform (2012), http://www.marketcetera.com/site/

12. Giraph (2013), http://incubator .apache.org/giraph/

13. Hadoop (2013), http://hadoop.apache.org

14. Hedwig (2013), https://cwiki.apache.org/ZOOKEEPER/hedwig.html

15. Kirsch, J., Amir, Y.: Paxos for Systems Builders (2008), http://www.cnds.jhu.edu/pub/papers/cnds-2008-2.pdf

16. Ousterhout, J., et al.: The Case for RAMCloud. In: CACM (2011)

17. Ostrowski, K., Sakoda, C., Birman, K.: Self-replicating Objects for Multicore Platforms. In: D'Hondt, T. (ed.) ECOOP 2010. LNCS, vol. 6183, pp. 452-477. Springer, Heidelberg (2010)

18. Ostrowski, K., Birman, K., Dolev, D.: Quicksilver Scalable Multicast (QSM). In: NCA 2008 (2008)

19. Ostrowski, K., Birman, K., Dolev, D., Ahnn, J.H.: Programming with Live Distributed Objects. In: Vitek, J. (ed.) ECOOP 2008. LNCS, vol. 5142, pp. 463-489. Springer, Heidelberg (2008)

20. Jayaram, K.R.: Elastic Remote Methods. Technical Report (2013), http://www. jayaramkr.com/elasticrmi

21. LibPaxos (2013), http://libpaxos.sourceforge.net/

22. Memcached (2013), http://www.memcached.org

23. Escriva, R., Wong, B., Sirer, E.: HyperDex: a Distributed, Searchable Key-Value Store. In: SIGCOMM 2012 (2012)

24. SPEC Open Systems Group (OSG). Report on Cloud Computing to the OSG Steering Committee (2012), http://www.spec.org/osgcloud/docs/osgcloudwgreport20120410.pdf

25. Zookeeper (2013), http://zookeeper.apache.org/ 